\title{
The early afterglow
}

\section{R. Sari ${ }^{1}$ and T. Piran ${ }^{2}$}

1 Theoretical Astrophysics, California Institute of Technology, Pasadena, CA 91125, U.S.A.

2 Racah Institute of Physics, The Hebrew University, Jerusalem 91904, Israel, and Department of Physics, Columbia University, New York, NY 10027, U.S.A.

Received December 29; accepted December 29, 1998

\begin{abstract}
We calculate the expected spectrum and light curves of the early afterglow. For short GRBs the peak of the afterglow will be delayed, typically, by few dozens of seconds after the burst. The X-ray and $\gamma$-ray characteristics of this delayed emission provide a way to discriminate between late internal shocks emission (part of the GRB) and the early afterglow signal. Detection of this delayed emission will prove the internal shock scenario as producing the GRB, and will pinpoint the initial Lorentz factor $\gamma_{0}$. In the optical band, the dominant emission arises from the reverse shock. This shock, carries a comparable amount of energy to the forward shock. It radiates this energy at much lower frequencies, producing a short optical flash of 15th magnitude or brighter.
\end{abstract}

\section{Introduction}

In the internal-external scenario, the GRB is produced by internal shocks while the afterglow is produced by the interaction of the flow with the ISM. The original fireball model was invoked to explain the Gamma-Ray Bursts (GRBs) phenomena. It requires extreme relativistic motion, with a Lorentz factor $\gamma>100$.

The afterglow observations, which fit the theory rather well, are considered as a confirmation of the fireball model. However, the current afterglow observations, which detect radiation from several hours after the burst onwards, do not probe the initial extreme relativistic conditions. By the time of the present observations, several hours after the burst, the Lorentz factor is less than $\sim 10$, and it is independent of the initial Lorentz factor.

Afterglow observations, a few seconds after the burst, can provide the missing information concerning the initial phase and prove the internal shock scenario. Such rapid observations are possible, in principle, with future missions (Kulkarni \& Harrison, private communication).

\section{The forward shock}

The synchrotron spectrum from relativistic electrons that are continuously accelerated into a power law energy dis- tribution is always given by four power law segments, separated by three critical frequencies: $\nu_{\mathrm{sa}}$ the self absorption frequency, $\nu_{\mathrm{c}}$ the cooling frequency and $\nu_{\mathrm{m}}$ the characteristic synchrotron frequency.

Using the relativistic shock jump condition and assuming that the electrons and the magnetic field acquire fractions $\epsilon_{\mathrm{e}}$ and $\epsilon_{\mathrm{B}}$ of the equipartition energy, we obtain:

$\nu_{\mathrm{m}}=1.110^{19} \mathrm{~Hz}\left(\frac{\epsilon_{\mathrm{e}}}{0.1}\right)^{2}\left(\frac{\epsilon_{\mathrm{B}}}{0.1}\right)^{1 / 2}\left(\frac{\gamma}{300}\right)^{4} n_{1}^{1 / 2}$

$\nu_{\mathrm{c}}=1.110^{17} \mathrm{~Hz}\left(\frac{\epsilon_{\mathrm{B}}}{0.1}\right)^{-3 / 2}\left(\frac{\gamma}{300}\right)^{-4} n_{1}^{-3 / 2} t_{\mathrm{s}}^{-2}$,

$F_{\nu, \max }=220 \mu \mathrm{Jy} \mathrm{D}_{28}^{-2}\left(\frac{\epsilon_{\mathrm{B}}}{0.1}\right)^{1 / 2}\left(\frac{\gamma}{300}\right)^{8} n_{1}^{3 / 2} t_{\mathrm{s}}^{3}$

$\nu_{\mathrm{sa}}=220 \mathrm{GHz}\left(\frac{\epsilon_{\mathrm{B}}}{0.1}\right)^{6 / 5}\left(\frac{\gamma}{300}\right)^{28 / 5} n_{1}^{9 / 5} t_{\mathrm{s}}^{8 / 5}$.

These scalings generalize the adiabatic scalings obtained by Sari et al. (1998) to an arbitrary hydrodynamic evolution of $\gamma(t)$.

For typical parameters, during the early afterglow $\nu_{\mathrm{c}}<\nu_{\mathrm{m}}$, so fast cooling occurs. The spectrum of fast cooling electrons is described by four power laws: (i) For $\nu<\nu_{\mathrm{sa}}$ self absorption is important and $F_{\nu} \propto \nu^{2}$. (ii) For $\nu_{\mathrm{sa}}<\nu<\nu_{\mathrm{c}}$ we have the synchrotron low energy tail $F_{\nu} \propto \nu^{1 / 3}$. (iii) For $\nu_{\mathrm{c}}<\nu<\nu_{m}$ we have the electron cooling slope $F_{\nu} \propto \nu^{-1 / 2}$. (iv) For $\nu>\nu_{\mathrm{m}} F_{\nu} \propto \nu^{-p / 2}$, where $p$ is the index of the electron power law distribution.

In the early afterglow, the Lorentz factor is initially constant. After that the evolution can be of two types (Sari 1997). Thick shells, which corresponds to long bursts, begin to decelerate with $\gamma(t) \sim t^{-1 / 4}$. Only later there is a transition to deceleration with $\gamma(t) \sim t^{-3 / 8}$. The light curves for such bursts can be obtained by substituting these scalings in Eqs. (1-4). However, the complex internal shocks GRB signal would overlap, for these long bursts, the smooth external shock afterglow signal. The separation of the observations to GRB and early afterglow would be rather difficult. 


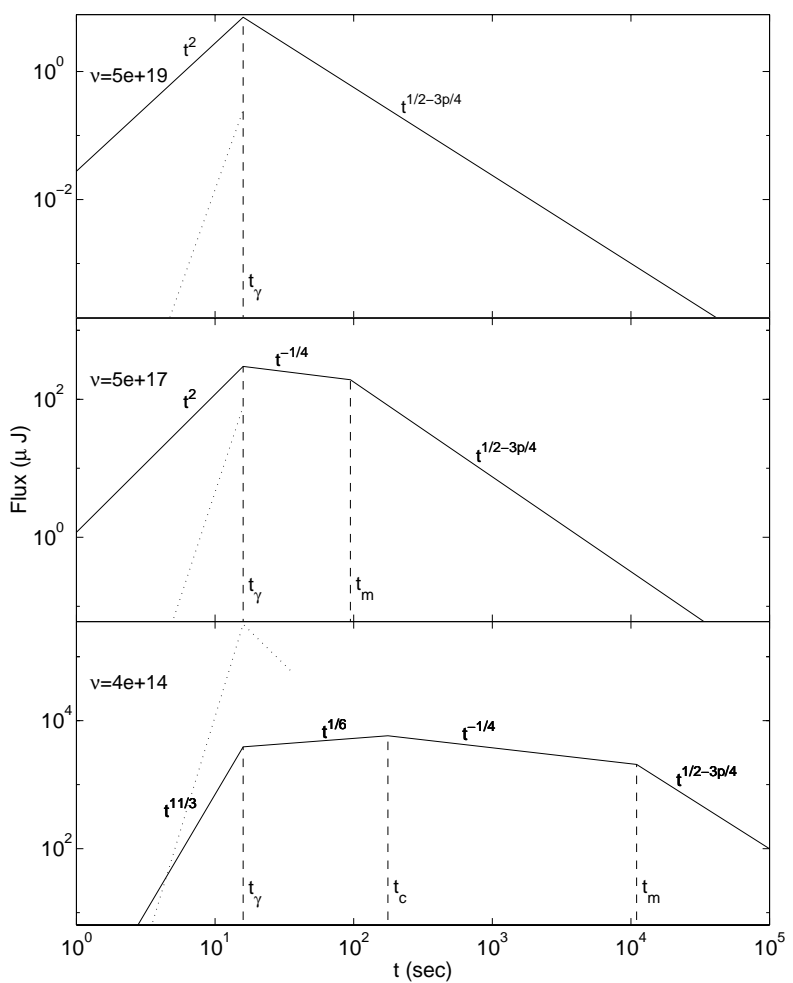

Fig. 1. Light curves of the forward (solid) and reverse (dashed) shocks in three energy bands

For thin shells, that correspond to short bursts, there is no intermediate stage of $\gamma(t) \sim t^{-1 / 4}$. There is a single transition, at the time $t_{\gamma}=\left(3 E / 32 \pi \gamma_{0}^{8} n m_{\mathrm{p}} c^{5}\right)^{1 / 3}$, from a constant velocity to self-similar deceleration with $\gamma(t) \sim t^{-3 / 8}$. The possible light curves are illustrated in Fig. 1. As the intial afterglow peaks several dozen seconds after the GRB there should be no difficulty to detect it.

The detection of a delayed emission which fits the light curves of Fig. 1, would enable us to determine $t_{\gamma}$. Using $t_{\gamma}$ we could proceed to estimate the initial Lorentz factor: $\gamma_{0}=240 E_{52}^{1 / 8} n_{1}^{1 / 8}\left(t_{\gamma} / 10 \mathrm{~s}\right)^{-3 / 8}$.

If the second peak of GRB 970228, delayed by $35 \mathrm{~s}$, is indeed the afterglow rise, then $\gamma_{0} \sim 150$ for this burst.

\section{The reverse shock and the optical flash}

There are many attempts to detect early optical emission and there is a good chance that this emission will be observed in the near future. A strong 5th magnitude optical flash would have been produced if the fluence of a moderately strong GRBs, $10^{-5} \mathrm{erg} / \mathrm{s} / \mathrm{cm}^{2}$ would have been released on a time scale of $10 \mathrm{~s}$ in the optical band. Even a small fraction of this will be easily observed. It is important, therefore, to explore the expected optical emission from the GRB and the early afterglow.

During the GRB and the initial emission from the forward shock the emission peaks in $\gamma$-rays, and only extremely small fraction is emitted in the optical band. For example, the prompt optical flash from the GRB would be of 21st magnitude if the flux drops according to the synchrotron low energy tail of $F_{\nu} \sim \nu^{1 / 3}$.

A considerably stronger flux is obtained from the reverse shock. The reverse shock contains, at the time it crosses the shell, a comparable amount of energy to the forward shock. However, its effective temperature is significantly lower (typically by a factor of $\gamma \sim 300$ ) than that of the forward shock. The resulting peak frequency is therefore lower by $\gamma^{2} \sim 10^{5}$. A more detailed calculation shows that the reverse shock frequency is

$\nu_{\mathrm{m}}=1.210^{14} \mathrm{~Hz}\left(\frac{\epsilon_{\mathrm{e}}}{0.1}\right)^{2}\left(\frac{\epsilon_{\mathrm{B}}}{0.1}\right)^{1 / 2}\left(\frac{\gamma_{0}}{300}\right)^{2} n_{1}^{1 / 2}$.

The cooling frequency is similar to that of the forward shock, since both have the same magnetic field and the same Lorentz factor. Using the parameters obtained by Granot et al. (1998), from the afterglow of GRB 970508, and using $\gamma_{0}=300$ we get for the reverse shock $\nu_{\mathrm{c}}=$ $310^{16} \mathrm{~Hz}$ and $\nu_{\mathrm{m}}=310^{14} \mathrm{~Hz}$ leading to an 8th magnitude flash. With higher initial Lorentz factor of $\gamma_{0}=10^{4}$ the flash drops to 13th magnitude. Inverse Compton cooling, if exists, can reduce the flux by $\sim 2$ magnitudes, while self absoption can influence only very short bursts with small surface area. Therefore, quite concervatively, the optical flash should be stronger than 15th magnitude and should be soon seen with modern experiments. The reverse shock signal is very short living. After the shock crosses the shell, no new electrons are injected and there is no emission above $\nu_{\mathrm{c}}$. Moreover, $\nu_{\mathrm{c}}$ drops fast with time as the shell's material cools adiabatically.

\section{Discussion}

The early afterglow multi-wavelength radiation could provide interesting and invaluable information on the extreme relativistic conditions that occur at this stage. The emission from the forward shock, which continues later as the observed afterglow signals is in the $\gamma$-rays or X-rays. The reverse shock emission could have a strong, short living, optical component which we expect to be brighter than 15 th magnitude. Some of the current basic ideas concerning the fireball models should be revised if such signals will not be seen by new detectors that should become operational in the near future.

Acknowledgements. This research was supported by the USIsrael BSF 95-328 and by a grant from the Israeli Space Agency. R.S. thanks the Sherman Fairchild Foundation for support.

\section{References}

Granot J., Piran T., Sari R., 1998, astro-ph/9808007

Park H.S., 1997, ApJ 490, L21

Sari R., 1997, ApJ 489, L37

Sari R., Piran T., Narayan R., 1998, ApJ 497, L17

Sari R., Piran T., 1999 (submitted) 